PROCEEDINGS OF THE

AMERICAN MATHEMATICAL SOCIETY

Volume 133, Number 4, Pages 1223-1230

S 0002-9939(04)07611-7

Article electronically published on October 15, 2004

\title{
RELATIVE FLUX HOMOMORPHISM IN SYMPLECTIC GEOMETRY
}

\author{
YILDIRAY OZAN
}

(Communicated by Jon G. Wolfson)

\begin{abstract}
In this work we define a relative version of the flux homomorphism, introduced by Calabi in 1969, for a symplectic manifold. We use it to study (the universal cover of) the group of symplectomorphisms of a symplectic manifold leaving a Lagrangian submanifold invariant. We also show that some quotients of the universal covering of the group of symplectomorphisms are stable under symplectic reduction.
\end{abstract}

\section{INTRODUCTION}

In 1969 Calabi introduced the flux homomorphism for a symplectic manifold $(M, \omega)([1])$. Let $\operatorname{Symp}_{0}(M, \omega)$ denote the path component of the Lie group of symplectomorphisms of $(M, \omega)$ containing the identity. Also, let $\widetilde{\operatorname{Symp}}_{0}(M, \omega)$ denote the universal covering space of $\operatorname{Symp}_{0}(M, \omega)$. Note that an element of $\widetilde{\operatorname{Symp}_{0}}(M, \omega)$ is represented by a homotopy class of a smooth path $\psi_{t}$ in $\operatorname{Symp}_{0}(M, \omega)$ connecting the identity $\psi_{0}=i d$ to $\psi=\psi_{1}$, where the homotopies fix the end points of the paths. We will denote the homotopy class of $\psi_{t}$ by $\left\{\psi_{t}\right\} \in \widetilde{\operatorname{Symp}}_{0}(M, \omega)$. Any such $\psi_{t}$ determines a smooth family of vector fields $X_{t}: M \rightarrow T_{*} M$ defined by the formula

$$
\frac{d}{d t} \psi_{t}=X_{t} \circ \psi_{t}
$$

Now the flux homomorphism Flux : $\widetilde{\operatorname{Symp}}_{0}(M, \omega) \rightarrow H^{1}(M, \mathbb{R})$ is defined, for any $\left\{\psi_{t}\right\} \in \widetilde{\operatorname{Symp}}_{0}(M, \omega)$, as

$$
\operatorname{Flux}\left(\left\{\psi_{t}\right\}\right)=\int_{0}^{1}\left[\imath\left(X_{t}\right) \omega\right] d t .
$$

For connected $M$ identifying $H^{1}(M, \mathbb{R})$ with $\operatorname{Hom}\left(\pi_{1}(M), \mathbb{R}\right)$ the cohomology class Flux $\left(\left\{\psi_{t}\right\}\right)$ corresponds to the homomorphism $\pi_{1}(M) \rightarrow \mathbb{R}$ defined by

$$
\gamma \mapsto \int_{0}^{1} \int_{0}^{1} \omega\left(X_{t}(\gamma(s), \dot{\gamma}(s))\right) d s d t
$$

for any smooth loop $\gamma: S^{1} \rightarrow M$.

Received by the editors October 18, 2003 and, in revised form, December 13, 2003.

2000 Mathematics Subject Classification. Primary 53D22, 53D12; Secondary 53D20.

Key words and phrases. Symplectic manifold, Lagrangian submanifold, symplectomorphism, flux homomorphism. 
In the next section, we will define a relative flux homomorphism in the presence of a Lagrangian submanifold $L \subseteq M$, mainly following both the treatment and the notation of Section 10 of [4]. Most of the results and proofs of this section are analogous to those of [4] and we will omit the proofs of some of them unless the proof includes some new ingredients or makes the text more comprehensible. In the third section, we will show that some quotients of $\widehat{\operatorname{Symp}}_{0}(M, \omega)$ are stable under symplectic reduction.

\section{Relative FluX HOMOMORPhism}

Throughout this section, unless stated otherwise, $(M, \omega)$ is a connected closed symplectic manifold and $L$ a closed Lagrangian submanifold.

Let $\operatorname{Symp}(M, L, \omega)$ denote the $\operatorname{subgroup~of~} \operatorname{Symp}(M, \omega)$ consisting of symplectomorphisms leaving the Lagrangian submanifold $L$ invariant and $\operatorname{Symp}_{0}(M, L, \omega)$ the path component of $\operatorname{Symp}(M, L, \omega)$ containing the identity.

Remark 2.1. In general, $\operatorname{Symp}(M, L, \omega) \cap \operatorname{Symp}_{0}(M, \omega)$ is not path connected. Let $S^{2}$ be the unit sphere in $\mathbb{R}^{3}$ equipped with the standard symplectic form and $S^{1}$ be the intersection of $S^{2}$ with the $x y$-plane. Then $S^{1}$ is a Lagrangian submanifold of $S^{2}$. Let $\psi_{t}, t \in[0,1]$, denote the rotation of $\mathbb{R}^{3}$ about the $x$-axis by $t \pi$ radians and $\psi=\psi_{1}$. Since $\psi_{\mid S^{1}}$ is orientation-reversing, $\psi$ is not in $\operatorname{Symp}_{0}(M, L, \omega)$, even though it lies in $\operatorname{Symp}(M, L, \omega) \cap \operatorname{Symp}_{0}(M, \omega)$ trivially. Hence, $\operatorname{Symp}(M, L, \omega) \cap$ $\operatorname{Symp}_{0}(M, \omega)$ is not path connected.

Identifying the homotopy class of the constant loop at the identity element of the group $\operatorname{Symp}_{0}(M, L, \omega)$ with that of $\operatorname{Symp}_{0}(M, \omega)$, we obtain a canonical map from $\widehat{\operatorname{Symp}}_{0}(M, L, \omega)$ onto a subgroup of $\widehat{\operatorname{Symp}}_{0}(M, \omega)$, the subgroup of homotopy classes $\left\{\psi_{t}\right\}$ in $\widehat{\operatorname{Symp}}_{0}(M, \omega)$, where each $\psi_{t}$ leaves $L$ invariant. Then we have the following result.

Lemma 2.2. The flux homomorphism is well defined on $\widetilde{\operatorname{Symp}_{0}}(M, L, \omega)$ and takes values in the kernel of the restriction map $H^{1}(M, \mathbb{R}) \rightarrow H^{1}(L, \mathbb{R})$. If $L$ is connected, the flux homomorphism takes values in $H^{1}(M, L, \mathbb{R})$. Moreover, in this case the flux homomorphism

is onto.

$$
\text { Flux : } \widetilde{\operatorname{Symp}}_{0}(M, L, \omega) \rightarrow H^{1}(M, L, \mathbb{R})
$$

Proof. The flux homomorphism is well defined on $\widetilde{\operatorname{Symp}}_{0}(M, \omega)$ since homotopic loops in $\operatorname{Symp}_{0}(M, \omega)$ have the same value under the flux homomorphism (see Lemma 10.7 of [4]). Therefore, homotopic loops in $\operatorname{Symp}_{0}(M, L, \omega)$ have the same value, and hence the flux homomorphism is well defined on $\widehat{\operatorname{Symp}}_{0}(M, L, \omega)$.

Let $\left\{\psi_{t}\right\} \in \widehat{\operatorname{Symp}}_{0}(M, L, \omega)$ and $X_{t}$ the vector field defined by

$$
\frac{d}{d t} \psi_{t}=X_{t} \circ \psi_{t}
$$

Since $\psi_{t}$ leaves $L$ invariant, for any $p \in L$ we have $X_{t}(p) \in T_{p} L$. Note that to prove the first assertion it suffices to show that for any smooth loop $\gamma: S^{1} \rightarrow L$ the integral

$$
\int_{0}^{1} \int_{0}^{1} \omega\left(X_{t}(\gamma(s), \dot{\gamma}(s))\right) d s d t=0
$$


However, this trivially holds since both $X_{t}(\gamma(s))$ and $\dot{\gamma}(s)$ lie in $T_{\gamma(s)} L$, and $L$ is a Lagrangian submanifold.

Now suppose that $L$ is connected. Then the exact sequence

$$
\rightarrow H^{0}(M, \mathbb{R}) \stackrel{\sim}{\rightarrow} H^{0}(L, \mathbb{R}) \stackrel{0}{\rightarrow} H^{1}(M, L, \mathbb{R}) \rightarrow H^{1}(M, \mathbb{R}) \rightarrow H^{1}(L, \mathbb{R}) \rightarrow
$$

implies that the flux homomorphism takes values in the isomorphic image of the relative cohomology group $H^{1}(M, L, \mathbb{R})$ in $H^{1}(M, \mathbb{R})$.

To show surjectivity let $u \in \Omega^{1}(M)$ be a closed 1 -form that is exact on $L$. Then $u_{\mid L}=d h$ for some smooth function $h: L \rightarrow \mathbb{R}$. We can extend $h$ first to a tubular neighborhood and then to the whole of $M$. Replacing $u$ by $u-d h$ we can assume that $u_{\mid L}=0$. The closed 1-form $u$ gives a symplectic vector field $X_{t}$ on $M$, which is indeed constant in time. If $\psi_{t}$ denotes the smooth 1-parameter family of symplectomorphisms generated by $X_{t}$, then clearly

$$
\operatorname{Flux}\left(\left\{\psi_{t}\right\}\right)=\int_{0}^{1}\left[\imath\left(X_{t}\right) \omega\right] d t=\int_{0}^{1}[u] d t=[u] .
$$

We need to show that $\psi_{t}$ leaves $L$ invariant. Let $p \in L$ and $Y \in T_{p} L$ be any vector. Then by the construction of $u$ we have $0=u(p)(Y)=\imath\left(X_{t}\right) \omega(p)(Y)=\omega\left(X_{t}(p), Y\right)$ for any $Y \in T_{p} L$, which implies that $X_{t}(p) \in T_{p} L$, because $L$ is a Lagrangian submanifold. This finishes the proof.

The following theorem is the relative version of Theorem 10.12 of [4, which describes the kernel of the flux homomorphism. First we define the Hamiltonian symplectomorphisms of $M$ leaving $L$ invariant, denoted $\operatorname{Ham}(M, L)$, as the subgroup of $\operatorname{Symp}(M, L)$ consisting of symplectomorphisms $\psi$ such that there is a Hamiltonian isotopy $\psi_{t}:(M, L) \rightarrow(M, L), t \in[0,1]$, such that $\psi_{0}=i d$ and $\psi_{1}=\psi$; i.e., $\psi_{t}$ is a Hamiltonian isotopy of $M$ such that $\psi_{t}(L)=L$, for any $t \in[0,1]$.

Since $L$ is Lagrangian, a Hamiltonian $H_{t}: M \rightarrow \mathbb{R}$ generating the above isotopy is locally constant on $L$.

Theorem 2.3. Let $\psi \in \operatorname{Symp}_{0}(M, L, \omega)$. Then $\psi$ is a Hamiltonian symplectomorphism if and only if there exists a symplectic isotopy, $\psi_{t},[0,1] \rightarrow \operatorname{Symp}_{0}(M, L, \omega)$ such that $\psi_{0}=i d, \psi_{1}=\psi$ and $\operatorname{Flux}\left(\left\{\psi_{t}\right\}\right)=0$.

Moreover, if $\operatorname{Flux}\left(\left\{\psi_{t}\right\}\right)=0$, then $\left\{\psi_{t}\right\}$ is isotopic with fixed end points to a Hamiltonian isotopy through paths in $\operatorname{Symp}_{0}(M, L, \omega)$.

The proof of the above theorem is almost the same as that of Theorem 10.12 of [4, where one has to observe that the symplectomorphisms and isotopies of $M$ involved in the proof leave $L$ invariant.

In [4] the authors define the subgroup

$$
\Gamma(M)=\operatorname{Flux}\left(\pi_{1}\left(\operatorname{Symp}_{0}(M)\right)\right) \subseteq H^{1}(M, \mathbb{R}) .
$$

Similarly, we define its relative version

$$
\Gamma(M, L)=\operatorname{Flux}\left(\pi_{1}\left(\operatorname{Symp}_{0}(M, L, \omega)\right)\right) \subseteq H^{1}(M, L, \mathbb{R})
$$

as the image of the fundamental group of the identity component of the relative symplectic group $\operatorname{Symp}_{0}(M, L, \omega)$ under the flux homomorphism. As $\Gamma(M)$ the group $\Gamma(M, L)$ is a subgroup of $H^{1}\left(M, P_{\omega}\right)$, where $P_{\omega}$ is the additive subgroup $[\omega] \cdot H_{2}(M, \mathbb{Z}) \subseteq \mathbb{R}$, and hence both $\Gamma(M)$ and $\Gamma(M, L)$ are countable.

To state the next lemma we need to recall the correspondence between symplectomorphisms of $(M, \omega)$ that are $C^{1}$-close to the identity and closed 1-forms on $M$ 
that are close to the zero form. Consider the symplectic manifold $(M \times M,(-\omega) \oplus \omega)$ whose diagonal $\Delta \subset M \times M$ is a Lagrangian submanifold, diffeomorphic to $M$. Then the Lagrangian neighborhood theorem implies that there is a symplectomorphism $\Psi: \mathcal{N}(\Delta) \rightarrow \mathcal{N}\left(M_{0}\right)$ between the neighborhoods of the Lagrangian submanifolds $\Delta \subset M \times M$ and $M_{0} \subseteq\left(T^{*} M, \omega_{\text {can }}\right)$, where $M_{0}$ is the zero section of the $T^{*} M$ equipped with the canonical symplectic form $\omega_{\text {can }}$, satisfying $\Psi^{*}\left(\omega_{\text {can }}\right)=(-\omega) \oplus \omega$ and $\Psi(q, q)=q$, for any $q \in M$. Then the correspondence

$$
(\psi: M \rightarrow M) \mapsto \sigma=\mathcal{C}(\psi) \in \Omega^{1}(M)
$$

is defined by

$$
\Psi(\operatorname{graph}(\psi))=\operatorname{graph}(\sigma)
$$

provided that $\psi \in \operatorname{Symp}(M)$ is sufficiently $C^{1}$-close to the identity.

Studying the proof of the existence of such a symplectomorphism $\Psi$, one realizes that $\Psi$ can be chosen so that symplectomorphisms of $M, C^{1}$-close to the identity, leaving a Lagrangian submanifold $L$ invariant correspond to closed 1-forms that equal zero on $T L$ (see Theorem 3.32 and Theorem 3.14 of [4]). Indeed, for the proof of the below relative version one has to note that a symplectomorphism, $C^{1}$ close to the identity, leaves $L$ invariant if and only if the various 1-forms involved in the proofs of Theorem 3.32 and Theorem 3.14 of [4] vanish on $(L \times L) \cap \Delta$ and the vector fields are parallel to $L \times L$.

Lemma 2.4. Assume the above notation. Then there is a symplectomorphism $\Psi: \mathcal{N}(\Delta) \rightarrow \mathcal{N}\left(M_{0}\right)$ satisfying $\Psi(q, q)=q$ for any $q \in M$, such that if $\psi:$ $(M, \omega) \rightarrow(M, \omega)$ is a symplectomorphism that is sufficiently $C^{1}$-close to the identity map, then $\psi$ leaves a Lagrangian submanifold $L$ invariant if and only if $\sigma_{\mid T_{q} L}=0$ for any $q \in L$.

Remark 2.5. The above lemma can be restated as follows: Let $L$ be a Lagrangian submanifold of a symplectic manifold $(M, \omega)$. Then there is a symplectomorphism $\Psi: \mathcal{N}(\Delta) \rightarrow \mathcal{N}\left(M_{0}\right)$ satisfying $\Psi(q, q)=q$ for any $q \in M$ that maps a neighborhood of $(L \times L) \cap \Delta$ in $L \times L$ onto a neighborhood of $L_{0}=L \cap M_{0}$ in the conormal bundle to $L_{0} \subseteq T^{*} M$, which is $\left\{v \in T_{q}^{*} M \mid q \in L, v_{\mid T_{q} L}=0\right\}$.

An immediate consequence of the above lemma is the following corollary.

Corollary 2.6. Let $\psi:(M, \omega) \rightarrow(M, \omega)$ and $\sigma=\mathcal{C}(\psi) \in \Omega^{1}(M)$ be as in the above lemma. Assume that $\psi$ leaves a Lagrangian submanifold $L$ invariant. Then for any $t \in[0,1]$, the symplectomorphism $\psi_{t}$, corresponding to the closed 1-form to, leaves $L$ invariant.

Now we can prove the following relative version of Lemma 10.16 of [4].

Lemma 2.7. If $\psi \in \operatorname{Symp}_{0}(M, L, \omega)$ is sufficiently $C^{1}$-close to the identity and $\sigma=\mathcal{C}\left(\psi_{t}\right) \in \Omega^{1}(M)$, then $\psi \in \operatorname{Ham}(M, L)$ if and only if $[\sigma] \in \Gamma(M, L)$.

Proof. Let $\psi_{t}, t \in[0,1]$, be the symplectic isotopy with $\mathcal{C}\left(\psi_{t}\right)=t \sigma$. Note that by the above corollary $\psi_{t} \in \operatorname{Symp}_{0}(M, L, \omega)$. Lemma 10.15 of [4] implies that $\operatorname{Flux}\left(\left\{\psi_{t}\right\}\right)=-[\sigma]$. Since $\psi$ is a Hamiltonian symplectomorphism, the path $\psi_{t}$ extends to a loop $[0,2] \mapsto \operatorname{Symp}_{0}(M, L, \omega)$, which is generated by Hamiltonian vector fields with end points $\psi_{0}=i d=\psi_{2}$. Moreover,

$$
\operatorname{Flux}\left(\left\{\psi_{t}\right\}_{0 \leq t \leq 2}\right)=\operatorname{Flux}\left(\left\{\psi_{t}\right\}_{0 \leq t \leq 1}\right)=-[\sigma],
$$

and hence $[\sigma] \in \Gamma(M, L)$. 
Conversely, let $[\sigma] \in \Gamma(M, L)$. Choose a loop $\psi_{t} \in \operatorname{Symp}_{0}(M, L, \omega)$ such that $\operatorname{Flux}\left(\left\{\psi_{t}\right\}\right)=-[\sigma]$. This extends to the interval $[0,2]$ by $\mathcal{C}\left(\psi_{t}\right)=[(t-1) \sigma]$ for $1 \leq t \leq 2$. Then the resulting path $\psi_{t}, t \in[0,2]$, has zero flux. Now by Theorem [2.3 this path can be deformed to a Hamiltonian isotopy via a homotopy with fixed end points. Therefore $\psi=\psi_{2}$ is a Hamiltonian isotopy.

Lemma 2.8. Every smooth path $\psi_{t} \in \operatorname{Ham}(M, L)$ is generated by Hamiltonian vector fields.

The proof of the above lemma is completely analogous to that of Proposition 10.17 of [4] and thus will be omitted. The following corollary, which is the relative version of Corollary 10.18 of [4, is the main result of this section.

Corollary 2.9. Let $(M, \omega)$ be a closed connected symplectic manifold and $L$ a closed connected Lagrangian submanifold.

i) There is an exact sequence of simply connected Lie groups

$$
1 \rightarrow \widetilde{\operatorname{Ham}}(M, L) \rightarrow \widetilde{\operatorname{Symp}_{0}}(M, L, \omega) \rightarrow H^{1}(M, L, \mathbb{R}) \rightarrow 0
$$

where $\widetilde{\operatorname{Ham}}(M, L)$ is the universal cover of $\operatorname{Ham}(M, L)$ and the third homomorphism is the flux homomorphism.

ii) There is an exact sequence of Lie algebras

$$
0 \rightarrow \mathbb{R} \rightarrow C^{\infty}(M, L) \rightarrow \chi(M, L, \omega) \rightarrow H^{1}(M, L, \mathbb{R}) \rightarrow 0 .
$$

Here the third map is $H \mapsto X_{H}$, the fourth map is $X \mapsto[\imath(X) \omega]$, while $C^{\infty}(M, L)$ denotes the algebra of smooth functions on $M$ that are constant on $L$ and $\chi(M, L, \omega)$ is the algebra of symplectic vector fields $X$ on $M$ such that $X(q) \in T_{q} L$ for any $q \in L$.

iii) The sequence of groups

$$
1 \rightarrow \pi_{1}(\operatorname{Ham}(M, L)) \rightarrow \pi_{1}\left(\operatorname{Symp}_{0}(M, L, \omega)\right) \rightarrow \Gamma(M, L) \rightarrow 0
$$

is exact.

iv) There is an exact sequence of groups

$$
1 \rightarrow \operatorname{Ham}(M, L) \rightarrow \operatorname{Symp}_{0}(M, L, \omega) \rightarrow H^{1}(M, L, \mathbb{R}) / \Gamma(M, L) \rightarrow 0
$$

where the third map is induced by the flux homomorphism.

Proof. By Lemma 2.8 every smooth path $\psi_{t} \in \operatorname{Ham}(M, L)$ that starts at the identity is a Hamiltonian isotopy and therefore has zero flux. This implies that $\widetilde{\operatorname{Ham}}(M, L) \subseteq \operatorname{ker}$ (Flux). On the other hand, by Theorem 2.3, if $\operatorname{Flux}\left(\left\{\psi_{t}\right\}\right)=0$, then the path $\psi_{t}$ is homotopic, with fixed end points, to a Hamiltonian isotopy, and hence $\left\{\psi_{t}\right\} \in \widetilde{\operatorname{Ham}}(M, L)$. Now, the first statement follows from the surjectivity of the flux homomorphism (see Lemma 2.2). The second statement is easy and indeed follows from the proof of Lemma 2.2 .

The only nontrivial part in the third statement is the fact that the homomorphism $\pi_{1}(\operatorname{Ham}(M, L)) \rightarrow \pi_{1}\left(\operatorname{Symp}_{0}(M, L, \omega)\right)$ is injective. To see this, it suffices to show that any path $[0,1] \rightarrow \widehat{\operatorname{Symp}}_{0}(M, L, \omega)$ with end points in $\widetilde{\operatorname{Ham}}(M, L)$ is isotopic with fixed end points to a path in $\widetilde{\operatorname{Ham}}(M, L)=\operatorname{ker}($ Flux $)$. However, this is just the parameterized version of the first statement.

The last statement is also obvious. 


\section{ApplicAtions AND STABILITY UNDER SYMPLECTIC REDUCTION}

Combining Part (i) of Corollary 2.9 with its absolute version

$$
0 \rightarrow \widetilde{\operatorname{Ham}}(M) \rightarrow \widetilde{\operatorname{Symp}_{0}}(M, \omega) \rightarrow H^{1}(M, \mathbb{R}) \rightarrow 0,
$$

Corollary 10.18 of [4], we derive the following corollaries.

Corollary 3.1. Suppose that $(M, \omega)$ and $L \subseteq M$ are as in Corollary 2.9. If the first cohomology group, $H^{1}(M, \mathbb{R})$, is trivial, then

$$
\widetilde{\operatorname{Ham}}(M)=\widetilde{\operatorname{Symp}_{0}}(M, \omega)
$$

and

$$
\widetilde{\operatorname{Ham}}(M, L)=\widetilde{\operatorname{Symp}}(M, L, \omega) .
$$

Corollary 3.2. Let $(M, \omega)$ be a closed connected symplectic manifold and $L \subseteq M$ a closed connected Lagrangian submanifold. If $\operatorname{ImH}^{1}(L, \mathbb{R})$ denotes the image of the restriction map $H^{1}(M, \mathbb{R}) \rightarrow H^{1}(L, \mathbb{R})$, then the flux homomorphism induces an exact sequence

$$
1 \rightarrow N(M, L) \rightarrow \widetilde{\operatorname{Symp}}_{0}(M, \omega) \rightarrow \operatorname{Im}^{1}(L, \mathbb{R}) \rightarrow 0,
$$

where $N(M, L)$ is the normal closure of the product of the image of the canonical map

and $\widetilde{\operatorname{Ham}}(M)$ in $\widetilde{\operatorname{Symp}_{0}}(M, \omega)$.

$$
\widetilde{\operatorname{Symp}}_{0}(M, L, \omega) \rightarrow \widetilde{\operatorname{Symp}}_{0}(M, \omega)
$$

Example 3.3. i) Let $L=S^{1} \subseteq S^{2}=M$, where $S^{2}$ is equipped with any symplectic form. Then by the above corollaries,

$$
\begin{aligned}
\widetilde{\operatorname{Ham}}(M) & =\widetilde{\operatorname{Symp}}_{0}(M, \omega), \\
\widetilde{\operatorname{Ham}}(M, L) & ={\widetilde{\operatorname{Symp}_{0}}}_{0}(M, L, \omega)
\end{aligned}
$$

and

$$
\widetilde{\operatorname{Symp}}_{0}(M, \omega)=N(M, L) \text {. }
$$

ii) Let $L=S^{1} \times\{p t\} \subseteq S^{1} \times S^{1}=M$, where $M$ is equipped with any symplectic form. Then

$$
\begin{gathered}
\widetilde{\operatorname{Symp}_{0}}(M, \omega) / \widetilde{\operatorname{Ham}}(M) \simeq \mathbb{R}^{2}, \\
\widetilde{\operatorname{Symp}_{0}}(M, L, \omega) / \widetilde{\operatorname{Ham}}(M, L) \simeq \mathbb{R}
\end{gathered}
$$

and

$$
\widetilde{\operatorname{Symp}}_{0}(M, \omega) / N(M, L) \simeq \mathbb{R} .
$$

iii) Again let $M=S^{1} \times S^{1}$, and let $L$ be any smoothly embedded nullhomotopic circle in $M$. Then

$$
\begin{gathered}
\widetilde{\operatorname{Symp}}_{0}(M, \omega) / \widetilde{\operatorname{Ham}}(M) \simeq \mathbb{R}^{2}, \\
\widetilde{\operatorname{Symp}_{0}}(M, L, \omega) / \widetilde{\operatorname{Ham}}(M, L) \simeq \mathbb{R}^{2}
\end{gathered}
$$

and

$$
\widetilde{\operatorname{Symp}}_{0}(M, \omega)=N(M, L) \text {. }
$$


3.1. Stability under symplectic reductions. The next result is about the stability of the quotients

$$
\widetilde{\operatorname{Symp}}_{0}(M, \omega) / \widetilde{\operatorname{Ham}}(M) \text { and } \widetilde{\operatorname{Symp}}_{0}(M / \omega) / N(M, L)
$$

under symplectic reduction.

Let $S^{1}$ act in a Hamiltonian fashion on the symplectic manifold $(M, \omega)$ and $L$ be a Lagrangian submanifold contained in a level set $\mu^{-1}(c)$ of the moment map, $\mu: M \rightarrow \mathbb{R}$, of the $S^{1}$-action. Assume that $L$ is $S^{1}$ invariant and the $S^{1}$-action on the level set $\mu^{-1}(c)$ is free. Let $M_{\text {red }}=\mu^{-1}(c) / S^{1}$ be the symplectic quotient with the symplectic structure $\omega_{\text {red }}$ and $L_{r e d}=L / S^{1}$, which is a Lagrangian submanifold of $\left(M_{\text {red }}, \omega_{\text {red }}\right)$. A theorem of $\operatorname{Li}([3])$ states that the fundamental groups and hence the first cohomology groups of $M, \mu^{-1}(c)$ and $M_{r e d}$ are all isomorphic under the canonical homomorphisms. On the other hand, if $p: L \rightarrow L_{r e d}$ denotes the quotient map, then we have the following result (see [5]).

Corollary 3.4. Let $M, M_{\text {red }}, L$ and $L_{r e d}$ be as above. Then the map

$$
p^{*}: \operatorname{Im}\left(H^{i}\left(M_{\text {red }}, \mathbb{Q}\right) \rightarrow H^{i}\left(L_{\text {red }}, \mathbb{Q}\right)\right) \rightarrow \operatorname{Im}\left(H^{i}(M, \mathbb{Q}) \rightarrow H^{i}(L, \mathbb{Q})\right)
$$

is onto for any $i$ and is an isomorphism for $i=1$.

The above arguments together with Corollary 3.2 imply the following stability result.

Corollary 3.5. Let $M, M_{\text {red }}, L$ and $L_{\text {red }}$ be as above. Then we have

$$
\widetilde{\operatorname{Symp}_{0}}(M, \omega) / \widetilde{\operatorname{Ham}}(M) \simeq \widetilde{\operatorname{Symp}_{0}}\left(M_{\text {red }}, \omega_{\text {red }}\right) / \widetilde{\operatorname{Ham}}\left(M_{\text {red }}\right)
$$

and

$$
\widetilde{\operatorname{Symp}}_{0}(M, \omega) / N(M, L) \simeq \widetilde{\operatorname{Symp}_{0}}\left(M_{\text {red }}, \omega_{\text {red }}\right) / N\left(M_{\text {red }}, L_{\text {red }}\right) .
$$

3.2. Real algebraic varieties and their complexifications. In this subsection we will mention a different kind of stability of the above groups for real algebraic varieties. Let $X$ be a nonsingular compact real algebraic variety with a nonsingular projective complexification $i: X \rightarrow X_{\mathbb{C}}$. Note that any two nonsingular projective complexifications of $X$ are always birationally isomorphic and thus have the same fundamental group and the same first cohomology group (see p. 494 of [2]). Clearly $X_{\mathbb{C}}$ carries a Kähler and hence a symplectic structure such that $X$ becomes a Lagrangian submanifold. So, by Corollary 10.18 of [4] (or just let $L$ be the empty set in Corollary 2.9(i)) the quotient group

$$
\widetilde{\operatorname{Symp}}_{0}\left(X_{\mathbb{C}}, \omega\right) / \widetilde{\operatorname{Ham}}\left(X_{\mathbb{C}}\right)
$$

is determined only by $X$ and hence is independent of the projective complexification $i: X \rightarrow X_{\mathbb{C}}$.

Define $K H_{i}(X, \mathbb{R})$ as the kernel of the induced homomorphism

$$
i_{*}: H_{i}(X, \mathbb{R}) \rightarrow H_{i}\left(X_{\mathbb{C}}, \mathbb{R}\right)
$$

and $\operatorname{Im} H^{i}(X, \mathbb{R})$ as the image of the induced homomorphism

$$
i^{*}: H^{i}\left(X_{\mathbb{C}}, \mathbb{R}\right) \rightarrow H^{i}(X, \mathbb{R}) .
$$

In [6] 17] it is shown that both $K H_{i}(X, \mathbb{R})$ and $\operatorname{ImH}^{i}(X, \mathbb{R})$ are independent of the projective complexification $i: X \rightarrow X_{\mathbb{C}}$ and thus are (entire rational) isomorphism 
invariants of $X$. Moreover, by Corollary [3.2, for any topological component $X_{0}$ of $X$ the quotient group

$$
\widetilde{\operatorname{Symp}}_{0}\left(X_{\mathbb{C}}, \omega\right) / N\left(X_{\mathbb{C}}, X_{0}\right)
$$

is independent of the smooth projective complexification $i: X \rightarrow X_{\mathbb{C}}$ and hence is determined only by $X$ or equally by $X_{0}$.

\section{REFERENCES}

1. E. Calabi, On the group of automorphisms of a symplectic manifold, Problems in Analysis (ed. R. Gunning), Princeton University Press, New Jersey, 1970. MR.50:3268

2. P. Griffiths, J. Harris, Principles of Algebraic Geometry, John Wiley \& Sons, Inc., New York, 1994. MR95d:14001

3. H. Li, $\pi_{1}$ of Hamiltonian $S^{1}$ manifolds, Proc. Amer. Math. Soc. 131 (2003), 3579-3582. MR2004b:53145

4. D. McDuff, D. Salamon, Introduction to symplectic topology, Oxford University Press, New York, 1997.

5. Y. Ozan, On cohomology of invariant submanifolds of Hamiltonian actions, preprint.

6. _ Homology of non orientable real algebraic varieties, preprint.

7. 3175. MR2002m:14048

Department of Mathematics, Middle East Technical University, 06531 Ankara, TURKEY

E-mail address: ozan@metu.edu.tr 